\title{
New insights of red light-induced development
}

András Viczián ${ }^{1 *}$, Cornelia Klose ${ }^{2 *}$, Éva Ádám ${ }^{1}$, Ferenc Nagy ${ }^{1,3 \#}$

$\left(\frac{10}{20}\right.$

${ }^{1}$ Institute of Plant Biology, Biological Research Centre, Temesvári krt.62, H-6726 Szeged, Hungary.

${ }^{2}$ Institute of Biology2/ Botany, University of Freiburg, Schänzlestrasse 1, D-79104 Freiburg,

Germany

${ }^{3}$ Institute of Molecular Plant Science, School of Biological Sciences, University of Edinburgh, Edinburgh EH9 3JH, UK

\# Author for correspondence:

Ferenc Nagy

Phone: 00-36-62599718

Fax: 00-36-62433434

E-mail: nagy.ferenc@brc.mta.hu

* These authors contributed equally.

\section{Running title:}

Novel molecular aspects of phytochrome B action

\section{Key words:}

phytochrome B, photomorphogenesis, phosphorylation, SUMO, dark reversion

\footnotetext{
This article has been accepted for publication and undergone full peer review but has not been through the copyediting, typesetting, pagination and proofreading process which may lead to differences between this version and the Version of Record. Please cite this article as doi: $10.1111 /$ pce. 12880
} 


\section{SUMMARY STATEMENT}

Phytochromes sense changes in the ratio and intensity of R and FR content of sunlight and by initiating/controlling a complex signaling network regulate nearly all aspect of plant growth and development. Recent research revealed exciting new aspects at molecular level how these photoreceptors function, uncovered the basic difference in the mode of action for the two major phytochrome species phyA and phyB and demonstrated that phyB is also function as thermosensor. This review summarizes and discusses the most important discoveries that opened new avenues for phytochrome-B related research

\section{ABSTRACT (133 words)}

The red/far-red light absorbing photoreceptors phytochromes regulate development and growth, and thus play an essential role in optimizing adaptation of the sessile plants to the ever changing environment. Our understanding of how absorption of a red/far-red photon by phytochromes initiates/modifies diverse physiological responses has been steadily improving. Research performed in the last five years has been especially productive, and led to significant conceptual changes about the mode of action of these photoreceptors. In this review we focus on the phytochrome B photoreceptor, the major phytochrome species active in light-grown plants. We discuss how its light-independent inactivation (termed dark/thermal reversion), post-translational modification, including ubiquitination, phosphorylation, sumoylation as well as heterodimerisation with other phytochrome species modify red-lightcontrolled physiological responses. Finally we discuss how photobiological properties of phyB enable this photoreceptor to function also as thermosensor. 


\section{INTRODUCTION}

Light is a key environmental factor affecting almost every aspect of plants' life. It is not only the main source of energy for photosynthesis, but also acts as a developmental clue to harmonize growth with the ambient light environment, a process termed photomorphogenesis. To alter the developmental program active in the dark (skotomorphogenesis) and thereby to ensure proper photomorphogenesis, plants have evolved a battery of photoreceptors. These sensors monitor the light spectrum, selectively absorb photons with different energies and translate light energy into biological signals to modulate the expression of thousands of genes that ultimately culminate in defined physiological responses. The widely used model plant Arabidopsis thaliana possesses the following photoreceptors: (i) the UV RESISTANCE LOCUS 8 (UVR8) absorbs ultraviolet B (Jenkins, 2014), (ii) the phototropins (Christie, 2007), the cryptochromes (Yu et al., 2010) and ZEITLUPE type receptors (Kim et al., 2007) are responsible for blue/UV-A perception, and (iii) phytochromes (phy) absorb red (R) and far-red (FR) light (Bae \& Choi, 2008; Franklin \& Quail, 2010).

Phytochromes exist in two interchangeable forms: the Pr form absorbs $\mathrm{R}$ light $\left(\lambda_{\max }=660 \mathrm{~nm}\right)$, whereas the Pfr form absorbs FR light $\left(\lambda_{\max }=730 \mathrm{~nm}\right)$. Phytochromes are synthesized in the Pr form in dark-grown seedlings, and absorption of a red photon induces conversion of Pr to Pfr, which is the biologically active phy conformer (Rockwell et al., 2006). Pfr is rapidly converted back to Pr by FR light (photoreversion) or, in the absence of light, by dark reversion, also called thermal relaxation, (Mancinelli, 1994). This interconversion property of phytochromes allows these photoreceptors to function as R/FRdependent molecular switches. The Arabidopsis phytochrome gene family contains five genes encoding phyA through phyE (Clack et al., 1994). They are classified according to their stability: the type I is light-labile (phyA), whereas the type II phytochromes are lightstabile (phyB-E). phyA is the dominant phytochrome of dark-grown (etiolated) seedlings, but its amount decreases rapidly upon illumination. Type II phytochromes are the prevalent phytochromes of light-grown plants; among them phyB is the most abundant (Hirschfeld et al., 1998; Sharrock \& Clack, 2002). In photobiological terms three modes of action have been identified for phytochromes. Low fluence responses (LFRs) are typical R/FR reversible responses mediated nearly exclusively by type II phytochromes. Very low fluence responses (VLFRs) are triggered by extremely low quantities of light, mediated by phyA and not 
photoreversible, whereas the high irradiance responses (HIRs) produced by prolonged exposure to high-intensity light can be mediated by phyA or phyB (Nagy \& Schafer, 2002).

\section{PHYTOCHROME REGULATED PHYSIOLOGICAL RESPONSES}

In Arabidopsis, phyA plays an important role in seedling establishment during the transition from skotomorphogenesis to photomorphogenesis. This and various other aspects of phyA signalling are discussed in the accompanying chapter in this issue. The switch to light-driven development, however, is not exclusively regulated by phyA. For example, regulation of germination and seedling de-etiolation (Su et al., 2015) is mediated, beside phyA (Shinomura et al., 1996), also by phyB and other type II phytochromes (Hennig et al., 2002; Dechaine et al., 2009; Lee et al., 2012; Jiang et al., 2016). The latter process results in the spectacular change of seedling morphology and manifests itself as inhibition of hypocotyl elongation, inducing opening of the cotyledon hook and expansion of the cotyledons (McNellis \& Deng, 1995; Franklin \& Quail, 2010; Kami et al., 2010). In a light-dominated environment the action of type II phytochromes regulates production of functional photosynthetic apparatus, promotes chloroplast development (Chen et al., 2010) alters photorespiration (Igamberdiev et al., 2014),contributes to stomata development (Casson \& Hetherington, 2014) and regulates stomata opening (Wang et al., 2010). Apart from these responses phytochromes regulate (i) gravitropic orientation of roots and hypocotyls (Kim et al., 2011; Hopkins \& Kiss, 2012) and (ii) development of rosette, branching and apical dominance (Finlayson et al., 2010; Franklin \& Quail, 2010), thus, in principle, define the architecture of adult plants (Figure 1A).

$\operatorname{Pr}$ and Pfr forms of phytochromes have overlapping absorption spectra, thus these photoreceptors are also able to monitor the R/FR ratio of sunlight. This is of particular importance in natural habitats, when light is reflected or filtered through the leaves of neighbouring plants. Under a dense canopy the R/FR ratio of sunlight can dramatically change, because chlorophylls and carotenoids efficiently absorb R but not FR light, which results in a low $\mathrm{R} / \mathrm{FR}$ ratio. Changes in $\mathrm{R} / \mathrm{FR}$ ratio drastically modulate phytochrome signalling and trigger the so-called shade avoidance syndrome (SAS). This response, characterized by specific morphological changes such as leaf hyponasty, increased apical dominance, elongated petioles and early flowering, is of great importance for plants as it is essential for overgrowing competitors to optimize the efficiency of photosynthesis (Casal, 2012; Casal, 2013; Fraser et al., 2016). SAS is mediated dominantly by phyB, but all members of the phy family are involved in the response, except for phyC (Franklin et al., 
2003). As stated above phyB as phyB Pfr primarily mediates plant growth and development in response to changes in $\mathrm{R} / \mathrm{FR}$ ratios and fluences in the ambient light environment. However, several lines of evidence indicate that phyB is also functioning under FR-HIR conditions when the majority of phyB molecules exist in their inactive Pr conformation. For example, it has been shown that seedlings overexpressing PHYB-GFP show pronounced etiolation phenotypes compared with the wild type counterparts under FR light (Wagner et al., 1996; Casal et al., 2000; Hennig et al., 2001). This response can also be observed without the presence of phyA thus phyB inhibition of phyA function, under these circumstances, is not mediated by the direct interaction of these photoreceptors. More recently, it was also demonstrated that phyB is required for the proper nuclear accumulation of COP1 and SPA1 in FR, indicating that phyB can modulate the intracellular distribution of signaling components required for proper FR signaling (Zheng et al., 2013). However, other factors such availability of nutrients (Short, 1999) also affect this response thus unravelling the precise molecular machinery for phyB action in FR will require further investigations.

Phytochromes, especially phyB, have also been shown to play a role in modulating signalling induced by biotic stress (herbivory) (Ballare, 2009), abiotic salinity (Carvalho et al., 2011) and drought stress (Gonzalez et al., 2012) and thermosensing (Franklin et al., 2014; Johansson et al., 2014; Quint et al., 2016). Two recent papers which will be discussed in detail in this review, revealed the molecular mechanism underlying the role of phyB in integrating light and temperature induced signalling and established phyB as a bona fide thermosensor (Jung et al., 2016; Legris et al., 2016). All above described developmental/growth/stress responses similar to timing of flowering (Valverde et al., 2004; Endo et al., 2013) are also regulated by the circadian clock. A direct link between the action of red light receptors and the circadian clock has been already established. On the one hand all phytochromes, dominantly phyB, mediates transmission of light signals to the core clock mechanism (Devlin \& Kay, 2000; Mas et al., 2003; Huang et al., 2016) on the other hand, most of the light-regulated processes are modulated by the clock, illustrating the complex mutual interactions of light and clock signalling pathways (Greenham \& McClung, 2015) (Figure 1A).

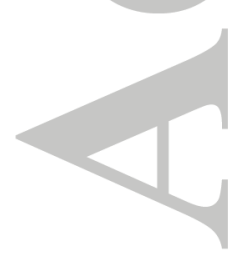

\section{STRUCTURE OF PHYTOCHROMES}

This article is protected by copyright. All rights reserved. 
All phytochromes have similar primary structures. The N-terminal domain of the apoprotein consists of the N-terminal extension (NTE), the PAS (PER-ARNT-SIM), the GAF (cGMP-specific phosphodiesterases, adenylyl cyclases and FhlA) and the PHY (phytochrome) domains (Figure 1B). The GAF domain cradles a linear tetrapyrrole chromophore (phytochromobilin) attached via a thioether bond to a conserved cysteine residue, and provides light sensitivity to the molecule (Nagatani, 2010). The C-terminal domain has regulatory functions, required for the dimerisation of the molecule; it contains two PAS domains as well as a motif related to histidine kinases (HKRD) (Nagatani, 2010; Vierstra \& Zhang, 2011). Expressing the N-terminal domain of type II phytochromes alone proved that this domain is essential for light perception and signal transduction (Matsushita $e t$ al., 2003; Oka et al., 2008; Adam et al., 2013). A recent report revealed the crystal structure of the $\mathrm{N}$-terminal domain of Arabidopsis phyB, and provided additional insights into the conformational change underlying phyB signalling (Burgie et al., 2014). The role of the different domains in mediating the interaction of phyB with signalling partners will be discussed in detail later in this review.

\section{MOLECULAR MECHANISMS OF PHYB SIGNALLING}

Light-induced translocation of phyB Pfr from the cytosol into the nucleus is an early and indispensable step in phyB signalling (Fankhauser \& Chen, 2008; Klose et al., 2015b). In contrast to phyA, which relies on the transport helper proteins FHY1 (FAR-RED ELONGATED HYPOCOTYL 1) and FHL (FHY1-LIKE), the mechanism of the lightdependent nuclear import of phyB is not comprehensively understood. PhyB nuclear import occurs independently of FHY1 and FHL (Hiltbrunner et al., 2006). The C-terminal half of phyB lacking the chromophore binding domain is localized in the nucleus independently of light (Sakamoto \& Nagatani, 1996; Matsushita et al., 2003). Further experiments demonstrated that intramolecular interactions between the $\mathrm{N}$-terminal and $\mathrm{C}$-terminal domains of phyB occur preferentially in the Pr form and are weakened in the Pfr form. Based on these observations a molecular mechanism has been proposed, in which the conformational transition from the Pr to the Pfr form unmasks the nuclear localization motif in the C-terminal domain to promote light-induced import of the photoreceptor into the nucleus (Chen et al., 2005). 
A more recent study offered an alternative interpretation of the above-mentioned findings. In a cell-free in vitro nuclear import system using isolated nuclei of the green alga Acetabularia, Pfeiffer et al. reconstituted the nuclear import of phyB only in the presence of transport factors that interact with phyB and carry an NLS (Pfeiffer et al., 2012). Interestingly, neither the full-length nor the N-terminal or C-terminal half of Arabidopsis phyB alone was able to accumulate in the Acetabularia nuclei, indicating that phyB itself does not contain a functional intrinsic NLS-motif. Addition of PIF3 (PHYTOCHROME INTERACTING FACTOR 3) to the system induced nuclear import of phyB as well as of both phyB fragments. PIF3 was previously shown to interact with both the $\mathrm{N}$ - and C-terminal halves of phyB, whereby binding to the N-terminal domain was Pfr-dependent (Ni et al., 1998; Ni et al., 1999). In the Acetabularia system PIF3-mediated nuclear import of the C-terminal phyB fragment occurred independently of light, whereas that of the $\mathrm{N}$-terminal fragment was clearly red-light-induced, indicating that the higher affinity of PIF3 to the Pfr-form is the reason for its light-dependent accumulation in the nucleus. The minimal requirements for a protein facilitating the nuclear import of phyB were narrowed down to a combination of a phyB-binding domain and an NLS, implying that any protein that interacts with phyB in a Pfr-specific fashion and contains an NLS could potentially mediate light-induced nuclear phyB import. This was further supported by the observation that nuclear import of phyB in vivo was impaired but not completely abolished in a pifq mutant lacking 4 of the PIF proteins (pifq $=$ pif1pif3pif4pif5), which indicates that proteins other than PIFs are involved in the nuclear translocation of phyB (Pfeiffer et al., 2012).

In the nucleus phyB controls seedling development by inhibiting two classes of repressors of photomorphogenesis: the COP1 (CONSTITUTIVELY PHOTOMORPHOGENIC1)/ SPA (SUPPRESSOR OF phyA-105) complex and the PHYTOCHROME INTERACTING FACTORS (PIFs). These repressors by acting synergistically promote skotomorphogenesis, but are inhibited by photoactivated phytochromes allowing photomorphogenic development in light. In darkness the E3 ubiquitin ligase COP1 forms complexes with members of the SPA (SPA1-SPA4 in Arabidopsis) and PIF families and targets positive regulators of photomorphogenic growth for degradation by the proteasome (Xu et al., 2014). Phytochromes inactivate the COP1/SPA/PIF complex leading to exclusion of COP1 from the nucleus, resulting in stabilization of its target proteins (Osterlund \& Deng, 1998; Subramanian et al., 2004; Pacin et al., 2014) and degradation/inactivation of PIFs (Al-Sady et al., 2006). However, until recently the molecular mechanism underlying COP1/SPA inactivation was not understood. It was demonstrated that 
phyA Pfr and phyB Pfr interact directly with SPA1, and by reorganizing the COP1/SPA complex they promote photomorphogenic development (Lu et al., 2015; Sheerin et al., 2015). These authors show that photoactivated phyB competes with COP1 for SPA binding, thereby disturbing the direct interaction between COP1 and SPA. Since SPA1 has been shown to enhance the E3 ubiquitin ligase activity of COP1 in the complex (Seo et al., 2003), it is not yet clear whether disruption of the COP1/SPA complex by phyB directly interferes with COP1 function on its target proteins, or rather eliminates the positive effect of SPA1 on COP1 activity. The finding that photoactivated phytochromes disrupt the direct interaction of COP1 and SPA provides a mechanistic model to explain the fast inactivation of the COP1/SPA complex independently of the slow process of COP1 exclusion from the nucleus.

Accumulation of phyB Pfr in the nucleus further initiates inactivation and degradation of PIFs that act as negative regulators of photomorphogenesis as well. PIFs are basic helixloop-helix (bHLH) type transcription factors that regulate gene expression to promote skotomorphogenesis (Duek \& Fankhauser, 2005; Leivar et al., 2008; Shin et al., 2009). Photoactivated phyB directly interacts with PIFs and induces their phosphorylation, ubiquitination and subsequent degradation by the proteasome (Al-Sady, et al., 2006; Shen et al., 2007; Shen et al., 2008; Leivar \& Quail, 2011; Ni et al., 2013). Recently, the in vivo phosphorylation sites of PIF3 have been determined during dark-to-light transition. Introducing multiple missense point mutations at the phosphorylation sites stabilized the protein in light, whereas phospho-mimic mutations promoted PIF3 degradation in the absence of light. These findings supported the conclusion that light-induced phosphorylation of PIF3 is indeed required for its subsequent degradation and for the negative feedback modulation of phyB levels by PIFs in prolonged light (Ni et al., 2013)

Recently Park et al. presented evidence that PIF degradation might not be the primary mechanism by which phytochromes inhibit these repressors of photomorphogenesis. The authors showed that the Pfr form of phyB was able to inhibit the DNA binding capacity of PIF3, thereby preventing association to its target promoters in vivo (Park et al., 2012). These data indicated that phyB inhibition of PIF function requires interaction of these proteins but mediated by two different mechanisms, i.e. sequestration of PIFs and/or stimulation of their degradation. In this aspect we note that a recent work showed that phyB signalling in one cell, can efficiently initiate PIF degradation in other cells that do not contain phyB. (Kim et al., 2016). This observation suggests that phyB initiated cell to cell signalling is involved in controlling activity of PIFs but (i) the chemical identity of the mobile signal(s), (ii) the 
molecular machinery mediating this type of degradation of PIF3 as well (iii) the overall impact of cell to cell communication on phyB signalling will remain to be elucidated.

Based on in vitro assays Martinez-Garcia et al. have proposed the hypothesis that light-dependent interaction with PIF3 recruits phyB to promoter elements of genomic targets, introducing the idea that phyB could be directly involved in the regulation of gene expression (Martinez-Garcia et al., 2000). On the one hand it has been shown that phyA was able to associate with genomic DNA at promoter elements of numerous genes, many of them were identified as phyA-regulated target gene (Chen et al., 2014). On the other hand a very recent report also demonstrated that phyB, similar to phyA can also be recruited to genomic promoter elements possibly via interaction with DNA-binding transcription factors (Jung et al., 2016). These data indicate that individual and selective modulation of gene expression by phyA and phyB could play an important role in light induced signalling.

\section{THE FUNCTIONAL ROLE OF DARK REVERSION IN PHYB SIGNALLING}

PhyB acts as a light quality and quantity sensor and gradually controls photomorphogenic development depending on the light conditions. Analyses of phyB overexpression lines demonstrated that the light sensitivity of phyB-mediated photomorphogenic responses depends on phyB abundance (Wagner et al., 1991; Rausenberger et al., 2010). More precisely, the number of physiologically active Pfr molecules quantitatively determines the signalling efficiency of phyB. Since the absorption spectra of $\mathrm{Pr}$ and $\mathrm{Pfr}$ overlap considerably, a dynamic photoequilibrium between the Pfr and the Pr forms is established depending on the wavelength. The Pfr form has a higher energy state than the Pr form and is thermally unstable. Thus relaxation of Pfr into Pr can occur in a light-independent fashion (therefore it is also termed dark reversion), but displays a strong temperature dependency (Schäfer \& Schmidt, 1974; Hennig \& Schäfer, 2001; Klose et al., 2015a). A fast dark reversion process is able to compete with the light reaction of Pr-to-Pfr formation under nonsaturating light conditions, leading to steady state Pfr levels lower than the photoequilibrium (the maximal relative Pfr level established depending on the light quality). Consequently, photoconversion and dark reversion determine the steady state level of the active Pfr conformation, enabling dynamic light quality and quantity sensing.

The PAS-GAF-PHY domains of Arabidopsis phyB N-terminal (photosensory module, PSM) recombinantly expressed in E. coli and reconstituted with phytochromobilin as chromophore exhibited efficient Pfr-to-Pr thermal reversion in vitro with a half-life of about 
$110 \mathrm{~min}$, indicating that dark reversion is a property of the phytochrome molecule (Zhang et al., 2013; Burgie et al., 2014). In contrast, dark reversion of full-length phyB expressed in yeast and reconstituted with phycocyanobilin as chromophore showed very rapid initial dark reversion, but did not revert completely back to Pr (Eichenberg et al., 2000; Sweere et al., 2001). More recent in vivo studies, however, revealed that phyB Pfr reverts almost completely to Pr within $4 \mathrm{~h}$ of darkness, corresponding to an overall half-life of $60 \mathrm{~min}$ (Sweere et al., 2001; Rausenberger et al., 2010; Klose et al., 2015a). Taken together, these studies indicate that in addition to being an intrinsic property of the phytochrome molecule, dark reversion is modulated by various external factors as well as intra- and intermolecular interactions.

Mutations altering conserved residues surrounding the chromophore in the phyB protein were shown to affect Pfr-to-Pr dark reversion differentially without impairing photoconversion. The Arg352Ala substitution stabilized Pfr against thermal reversion, whereas Arg322Ala caused a substantially faster dark reversion of purified recombinant PSM of phyB in vitro (Zhang et al., 2013). Arabidopsis phyB mutant seedlings expressing the fulllength phyB[Arg352Ala] showed normal phyB signalling under high fluence rates of red light and in white light, but were hypersensitive under low fluence rates, suggesting that thermal reversion impacts phyB action when light conditions are limiting. Consistent with this conclusion, Oka et al. showed that the Arg322Gln substitution reduced responsiveness of Arabidopsis seedlings expressing the full-length mutant variant under intermittent red light pulses (Oka et al., 2008).

The NTE domain of phyB has been shown to stabilize Pfr, and mutants lacking this domain exhibit accelerated dark reversion in vitro (Zhang et al., 2013). The PHY domain contains a unique tongue-like structure that interacts with the GAF domain bearing the chromophore. This protrusion has been implicated in the transmission of conformational changes from the chromophore retained in the GAF domain to the PHY domain and consequently the whole molecule. Thereby the tongue was found to refold during transmission from $\operatorname{Pr}$ to Pfr from a beta-strand to an alpha-helix (Takala et al., 2014). Mutations in this tongue region of the PHY domain of phyB, e.g. Arg582Ala, Gly564Glu (phyB-401) have been described leading to a dramatically enhanced thermal stability of the Pfr form resulting in strong hypersensitivity of seedlings grown under weak red light (Kretsch et al., 2000; Ádám et al., 2011; Zhang et al., 2013). In addition, the Glu812Lys mutation (phyB-101) in the second of the two PAS domains in the C-terminal of phyB (Figure 1B) caused enhanced dark reversion in combination with a loss-of-function 
phenotype, demonstrating that protein domains that are more distant from the chromophore could also affect Pfr thermal stability (Elich \& Chory, 1997). It would be interesting to investigate whether other phyB loss-of-function mutants might be affected in dark reversion as well.

Phytochromes form dimers in vivo, and dimerization has been shown to be important for their physiological function (Matsushita et al., 2003). Consequently, phytochrome dimers can exist in three different states: Pr-Pr, Pfr-Pr, and Pfr-Pfr. A recent study demonstrated that the different dimer species of phyB indeed exhibit differential kinetic properties that are fundamental for the mode of phyB action (Klose et al., 2015a). Already in 1987 it was proposed that dark reversion has different kinetics for Pfr-Pfr and Pfr-Pr dimers based on in vivo observations (Brockmann et al., 1987). This was supported by the finding that recombinant Pfr-Pr dimers expressed in yeast showed fast and complete dark reversion in contrast to Pfr-Pfr dimers that remained more stable (Hennig \& Schäfer, 2001). Klose et al. (2015a) combined in vivo measurements and mathematical modelling to demonstrate that PfrPr heterodimers and Pfr-Pfr homodimers exhibit extremely different dark reversion kinetics, with Pfr-Pr dark reversion being almost 100-fold faster as compared to Pfr-Pfr. These findings lead to the conclusion that in Arabidopsis the phyB Pfr-Pr heterodimer pool undergoes fast dark reversion, resulting in reduced amounts of active phyB, particularly under light conditions that favour the generation of Pfr-Pr heterodimers, e.g. lower light intensities or wavelengths above $690 \mathrm{~nm}$. As the physiological phyB function is inhibited under such light conditions, it was concluded that only the Pfr-Pfr homodimers in the nucleus are able to initiate phyB-mediated light signalling (Klose et al., 2015a). In other words, the slow dark reversion of the Pfr-Pfr homodimer determines the persistence of phyB signalling after transfer to darkness, whereas the extremely fast dark reversion of the Pfr-Pr heterodimer competes efficiently with the Pr to Pfr photoconversion, reducing the Pfr levels under nonsaturating irradiation.

The precise nature of the fast Pfr-Pr dark reversion process needs to be determined. It is possible that the thermal stability of the Pfr-Pr dimer is affected when only one of the two subunits has undergone the conformational change from Pr to Pfr. Alternatively, the Pfr form of phyB could be stabilized by interactions with other proteins, for example ARABIDOPSIS RESPONSE REGULATOR 4 (ARR4), and such stabilization may work more efficiently for the Pfr-Pfr homodimer (Sweere et al., 2001). Phosphorylation of specific amino acids, especially that of Ser86 residing in the N-terminal domain of phyB can also modify dark 
reversion and red light signalling by an ARR4-independent mechanism (Medzihradszky et al., 2013); this is discussed in more detail in the following section.

Upon light irradiation, phyB associates within discrete subnuclear structures named photobodies (PBs) (Chen et al., 2003; Fankhauser \& Chen, 2008). Light conditions establishing high Pfr levels promote the formation of large PBs in vivo (Trupkin et al., 2014; van Buskirk et al., 2014). Thus it has been proposed that these PBs function in stabilizing phyB Pfr, which allows phyB to continue controlling the level of PIFs and suppressing hypocotyl growth after light-dark transfer (Rausenberger et al., 2010; van Buskirk et al., 2014; Klose et al., 2015a). Very recently it was shown that PCH1 (PHOTOPERIODIC CONTROL OF HYPOCOTYL 1), a protein that is associated with the Evening Complex in Arabidopsis, binds phyB in a red-light-dependent manner and co-localizes with phyB into PBs (Huang et al., 2016). With the need to be verified experimentally, the authors presented a model, in which binding of $\mathrm{PCH} 1$ to phyB after light exposure slows dark reversion of phyB Pfr, thereby extending the lifetime of phyB-containing large PBs (Huang et al., 2016). A correlation between dark reversion rates, PB formation and stability has been observed previously: mutant phyB molecules exhibiting accelerated dark reversion often failed to localize to PBs under normal light conditions or required higher fluence rates of red light, whereas mutants with slower dark reversion accumulated into PBs even under weak fluence rates (Ádám et al., 2011; Medzihradszky et al., 2013; Zhang et al., 2013).

\section{POST-TRANSLATIONAL MODIFICATIONS OF PHYB}

\section{Ubiquitination}

The E3 ubiquitin ligase COP1 was shown to interact with the N-terminal fragment of phyB, it was capable to ubiquitinate the photoreceptor and ubiquination of phyB was stimulated by the presence of PIF3 in these in vitro assays (Jang et al., 2010). More recently, massspectrometry analysis of proteins co-purified with PIF3 from Arabidopsis identified components of a Bric-a-Brack/Tramtrack/Broad (BTB)-Cullin3-type E3 ubiquitin ligase as red-light-specific PIF3-interacting proteins (Ni et al., 2014). Interestingly, the two highly conserved BTB proteins LRB1 (Light-Response-BTB1) and LRB2 had been previously shown to be required for proteasomal phyB degradation (Christians et al., 2012) Ni et al., however, could show that PIF3 phosphorylation triggers recruitment of LRB E3 ubiquitin ligases to the PIF3-phyB complex, whereupon LRBs promote polyubiqutination and degradation of both PIF3 and phyB in vivo (Ni et al., 2014). The proposed PIF3-phyB co- 
degradation model provides a mechanistic explanation for phyB-induced PIF3 degradation and concurrent signal attenuation by photoreceptor degradation (Zhu \& Huq, 2014). PIF3 degradation is about 50 -fold faster as compared to phyB degradation. The strongly different degradation kinetics of PIF3 and phyB were explained by the different protein levels in seedlings, where phyB is much more abundant than PIF3, which was supported by the fact that overexpression of PIF3 enhanced phyB degradation (Ni et al., 2013; Ni et al., 2014). Whereas phyB degradation in red light was completely abolished in an lrb123 triple mutant, PIF3 degradation was only slowed down. The results are compatible with the hypersensitive phenotype of lrb123 in light (Christians et al., 2012) that is consistent with the observed higher phyB abundance in light, but not with a defective PIF3 degradation (Ni et al., 2014). These observations suggest that the main function of LRBs is signal attenuation by photoreceptor degradation, and that there is partial functional redundancy between the LRBs and other unknown E3 ligases for PIF3 degradation.

\section{Phosphorylation}

Early studies performed using purified oat and maize phytochromes indicated that phytochromes have autophosphorylation activity whereas sequence comparison showed that the C-terminal domain of phytochromes contains a region homologous to bacterial histidine kinases (Schneider-Poetsch et al., 1991). Research performed to clarify how and to what extent (reversible) phosphorylation modulates phyA action produced plenty of data (Kim et al., 2004; Ryu et al., 2005; Han et al., 2010), yet until very recently the significance of the postulated kinase activity of phyA (Yeh \& Lagarias, 1998; Fankhauser et al., 1999) was debated (for details see accompanying review article in this issue). Here we only note that a very recent report identified the kinase domains of various plant phytochrome species including oat and Arabidopsis phyA, and demonstrated that this region is critical for ATPbinding (Shin et al., 2016). These authors also provided convincing evidence that perturbation of this region inhibited phosphorylation of PIF3 by oat phyA in vitro, and confirmed in transgenic plants that the kinase activity of phyA is critical for efficient lightinduced signalling.

In contrast to phyA, our knowledge about the phosphorylation of phyB is rather limited, although it was shown that (i) PAPP5 and PAPP2c (PHYTOCHROMEASSOCIATED PROTEIN PHOSPHATASE) proteins bind to the Pfr form of phyB, (ii) their null mutants show reduced responses in $\mathrm{R}$ light, and that (iii) phyB is phosphorylated in vitro and also interacts with the protein phosphatase PAPPC2 (Ryu et al., 2005; Phee et al., 2008). These observations suggested that phosphorylation of the photoreceptor attenuates light 
signalling. More recent studies identified a number of phosphorylated residues of phyB (Medzihradszky et al., 2013; Nito et al., 2013). Medzihradszky et al. demonstrated that the Ser86 located in the N-terminal domain of the protein is phosphorylated in planta. The phospho-mimic phyB[Ser86Asp] mutant shows fast dark reversion, and thereby decreases the amount of phyB Pfr. The low Pfr level of the mutant phyB slows down the import of the receptor into the nucleus and limits its interaction with PIF3; in other words, phosphorylation of phyB effectively attenuates light signalling. Consistent with this conclusion the nonphosphorylatable phyB[Ser86Ala] mutant displays slower dark reversion in vitro and in planta, thus transgenic plants expressing this mutant exhibit hyperactive responses including inhibition of hypocotyl elongation, cotyledon expansion, shade avoidance and flowering, particularly under low light intensity conditions, where Pfr amount is limiting (Medzihradszky et al., 2013; Hajdu et al., 2015). Besides Ser86, work performed by Nito et al. revealed nine further phosphorylated amino acid positions in Arabidopsis phyB (Ser84, Tyr89, Tyr90, Tyr91, Ser94, Ser95, Tyr104, Ser106, Tyr113). These amino acids are located in a cluster named PCSM motif (Phosphorylation Cluster of Signaling Modulation) spanning from Ser84 to Tyr113 (Figure 1B) and are conserved evolutionarily, indicating their general regulatory importance (Nito et al., 2013). The phosphorylation of each identified amino acid negatively regulates phyB signalling, but among them Tyr104 has the most pronounced phenotype. Tyr104 is phosphorylated after light exposure, and the phospho-mimic mutant phyB[Tyr104Glu] possesses no light signalling activity at all, whereas the nonphosphorylated phyB[Tyr104Phe] shows enhanced activity as compared to wild-type phyB (Nito et al., 2013). Similarly to Ser86, phosphorylation of Tyr104 also attenuates phyB signalling, presumably also by accelerating dark reversion. These data suggest that this domain of the molecule could be a "hot-spot", where Pfr stability is regulated according to the actual light conditions.

Beside the PCSM domain, phyB was reported to be autophoshorylated at unknown sites within its NTE domain (1-100) by (Phee et al., 2008) in vitro and at the Ser596, Tyr601, Ser977, Ser1163 residues in planta (Nito et al., 2013). These latter amino acids were phosphorylated in the dark and in the light as well, and the function of these modifications is not known (Nito et al., 2013). A very recent study demonstrated that phyB and phyD similarly to phyA - have kinase activity, autophosphorylate and can phosphorylate PIF3 in vitro. The amino acids critical for ATP-binding reside in the N-terminal domain of phyA (1651) (Shin et al., 2016). The equivalent N-terminal domain of phyB appears to play a significant role in regulating dark reversion (see dark reversion chapter above). Thus we 
speculate, although the ATP-binding site and kinase activity of phyB is yet to be identified in planta, that modulation of dark reversion by reversible autophosphorylation and/or phosphorylation of phyB by other kinases as well its ability to phosphorylate other proteins must be harmonized.

\section{SUMOylation}

Reversible, covalent conjugation of Small Ubiquitin-Like Modifier (SUMO) molecules to target proteins regulates protein activity and different cellular responses in eukaryotic cells. The conjugation and removal of SUMO is performed by a small set of enzymes, which have conserved structure throughout different organisms (Miura \& Hasegawa, 2010; Hickey et al., 2012; Novatchkova et al., 2012). The sumoylation state of the protein pool depends on various factors (including stress, developmental state, hormonal signalling etc.), furthermore numerous plant SUMO substrates were identified in the past few years (Elrouby \& Coupland, 2010; Miller et al., 2010).

Recently it was reported that phyB is sumoylated in planta, the SUMOylated form of phyB accumulates to high levels when the receptor is in the Pfr form, and phyB SUMOylation is reversible (Sadanandom et al., 2015). It was also demonstrated that the target lysine of SUMO conjugation is located in the C-terminal domain of phyB. The sumoylation of the mutant phyB[Lys996Arg] is negligible, and the transgenic plants expressing this receptor are hypersensitive in $\mathrm{R}$ light. This phenotype could be - at least partly - explained by the reduced binding of the SUMOylated phyB to the negative regulator transcription factor PIF5. Thus these authors concluded that SUMOylation of phyB attenuates light signalling by reducing the formation/stability of the phyB-PIF complexes (Sadanandom et al., 2015). Consistent with its reversibility, the SUMOylation level of the phyB pool appears to be regulated at least partly by the concerted action of OVERLY TOLERANT TO SALT (OTS) 1 and 2 SUMO proteases. OTS1 binds directly to phyB and removes the SUMO from the protein. Compared to wild-type plants, the accumulation level of the SUMOylated phyB pool is higher in the otslots 2 mutant plants, which show a hyposensitive photomorphogenic phenotype in $\mathrm{R}$ light (Sadanandom et al., 2015). It remains to be seen if SUMOylation - similarly to phosphorylation - also targets, beside phyB, other phytochrome species and/or down-stream signalling components. 


\section{HETERODIMERIZATION OF TYPE II PHYTOCHROMES}

For many years, after discovering that phyA purified from dark-grown oat seedlings exists primarily as dimer (Lagarias \& Mercurio, 1985) it was generally agreed that the type II phytochromes are also active as homodimers. However, two seminal papers (Sharrock \& Clack, 2004; Clack et al., 2009) changed this view. First, these authors demonstrated that Arabidopsis contains multiple species of both homodimeric and heterodimeric phyB and phyD phytochromes, but phyA is present only as a homodimer and does not form heterodimers with any other phytochrome species. Next, they reported that phyC and phyE do not homodimerize, but heterodimerize with phyB and phyD and that the expression/activity of phyC in a phyBphyD mutant, where none of its dimerization partners was present, dropped dramatically (Clack et al., 2009). Clack et al. also showed that not only phyB but phyC and phyD, presumably as members of phyB/phyC and phyB/phyD heterodimers co-immunoprecipitate from seedling extracts with the PIF3 transcription factor in a R/FR-reversible manner (Clack et al., 2009). Although direct interaction of phyC, phyD and phyE with PIF3 has not yet been detected in planta, these results show that all phytochromes in homo- or heterodimeric forms appear to function through PIF-mediated pathways.

Two more recent reports demonstrated that (i) homodimers of the N-terminal fragments of all type II phytochromes were biologically active in the modulation of R-light-regulated photomorphogenesis (Adam et al., 2013) and that (ii) heterodimers of the N-terminal domains of phyB/phyC, phyB/phyD, phyB/phyC, phyB/phyE etc. generated by using a synthetic biological approach showed slightly different phenotypic responses when compared phyB/phyB. For example, the phyB/ phyB[Cys357Thr] heterodimer containing the chromophore-less version of phyB was active in petioles and cotyledons, but not in hypocotyls (Liu \& Sharrock, 2013). Taken together, the above findings suggested that the formation of such type II heteromeric photoreceptors increases the potential complexity of R/FR light sensing, for example phyC might signal only as heterodimer, yet the question of how and to what extent remained to be answered. Just recently by using a bottom-up assembly of phytochrome network Sanches-Lamas et a., provided more insight into the biological function of phytochrome heterodimerisation (Sanchez-Lamas et al., 2016). In this elegant study the authors first expressed each of the five phytochromes in the quintuple phyAphyBphyCphyDphyE mutant and then created lines expressing pairwise these phy genes in all possible combination. Analysis of this set of mutant plants revealed many new features 
of the phytochrome network and demonstrated among others that phyB alone is sufficient to confer full hypocotyl, germination responses to $\mathrm{R}$ and repress flowering but phyB and phyC co-action is needed to confer responsiveness to photoperiod. These findings indicate that phyB/phyB homodimers are mediating responses to light quality whereas phyB/phyC heterodimers are essential for the manifestation of a proper photoperiodic response. These authors also showed that association of phyB to nuclear bodies also modified by phyC and concluded that phyB/phyC heterodimers are probably active for longer periods in darkness which could be an important factor to repress flowering and hypocotyl elongation especially under short-day conditions. In addition, on the one hand they also clarified individual contribution of phyD and phyE to a variety of light controlled responses, for example they showed that phyE strongly repressed flowering but had little effect on controlling hypocotyl growth. On the other hand they also uncovered synergestic and antagonistic effects of phytochromes in controlling germination and flowering and hypothesized that at least part of these responses is mediated by heterodimers of the various phytochrome species. More importantly they have suggested by analysing a large number transgenic lines expressing these phytochromes at different level that the role of the individual phytochrome species is determined by the intrinsic properties of these photoreceptors (such as ability to heterodimerize, photochemical features, interaction with signaling partners etc.) rather than by the expression level or patterns. Nothwithstanding these very convincing data, however, it is also true that even a slight reduction of the phyB expression level significantly alters red light responsiveness, indicating that modification of the ratio of phyB/phyB homodimers by other type II phytochromes could be an important factor. At present, the molecular mechanism regulating/limiting homodimerization and/or heterodimerization of phyB with other type II phytochromes is not known, nor is it known how these phyB-containing heterodimers function, i.e. whether they regulate the expression of genes at least partly different from those regulated by homodimers. Given the importance of dark reversion and post-translational modifications of phyB in regulating red light-induced signalling, we speculate that these could also be affected by heterodimerization with phyC, phyD and phyE.

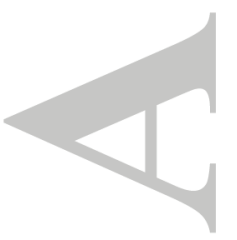




\section{ROLE OF PHYB IN TEMPERATURE SENSING/ INTEGRATION OF LIGHT AND TEMPERATURE SIGNALING}

A growing amount of findings has led to the recognition that light and temperature signals are integrated by multiple mechanisms (Franklin et al., 2014; Johansson et al., 2014; Quint et al., 2016). The morphological changes induced by high ambient temperature, collectively summarized as thermomorphogenesis, include the promotion of elongation growth which parallels the response to unfavourable light conditions in vegetational shade (Casal, 2012). Interestingly, PIF4, a positive regulator of the shade avoidance response, was identified as central component of ambient temperature signalling (Koini et al., 2009). PIF4 functions in regulating phytohormone biosynthesis and signalling. Expression of PIF4 is controlled by the circadian clock through repression by the Evening Complex but is increased by high temperature (Nozue et al., 2007; Nusinow et al., 2011). On the posttranslational level PIF4 activity and abundance is controlled by phyB. PIF4 interacts specifically with light activated phyB leading to its phosphorylation and subsequent degradation (Lorrain et al., 2008).

Two very recent complementary studies have demonstrated that phyB directly participates in temperature perception based on the temperature dependency of its kinetic properties (Jung et al., 2016; Legris et al., 2016). Although it has been described previously that dark reversion is strongly temperature dependent (Schäfer \& Schmidt, 1974; Hennig \& Schafer, 2001; Klose et al., 2015a) the two papers highlighted the role of dark reversion in plant temperature responses considering also the differential properties of the phyB dimers.

Jung et al. (2016) showed that high temperature accelerates the phyB Pfr decay during night time which is based on the temperature sensitivity of the slow dark reversion process of the Pfr-Pfr homodimer. Active phyB was shown to associate in a temperature dependent manner with promoters of genes that are also targeted by PIFs. Faster phyB dark reversion at higher temperature correlated with the loss of phyB occupancy at target gene promoters leading to the conclusion that phyB could transmit temperature information by inhibiting PIF activity through direct binding at target promoters. These findings were supported by extensive gene expression analyses showing that the warm temperature transcriptome is specifically affected by phytochrome activity during nighttime. Phytochrome null mutants displayed a constitutive warm temperature transcriptome even at low temperatures whereas in the constitutively active phyB[Tyr276His] allele the warm temperature transcriptome was constitutively repressed during night. 
Legris et al. (2016) showed that temperature regulation of phyB Pfr levels is effective not only at night but also during the day. In light, the steady state levels of phyB Pfr are determined by the photoconversion rates, depending on the light quality and intensity, as well as by the fast dark reversion rate of the Pfr-Pr heterodimer (Klose et al., 2015a). Using both, in vitro and in vivo spectroscopic assays, the authors demonstrated that the fast Pfr-Pr dark reversion rate of phyB is strongly sensitive to temperature (Legris et al., 2016). This is particularly obvious under low light conditions, where Pr to Pfr photoconversion is slower. Under such conditions the Pfr-Pr heterodimers are more abundant compared to higher light intensities and might undergo dark reversion rather than absorbing another photon to become Pfr-Pfr. High temperature favors the dark reversion reaction thereby reducing the Pfr steady state levels especially at low light conditions. PhyB containing nuclear bodies reflect the status of phyB since they are mainly composed of Pfr-Pfr homodimers. As a proxy for temperature effects on Pfr-Pfr levels Legris et al. (2016) quantified the nuclear body sizes of wild-type phyB and two phyB mutant alleles with suppressed thermal reversion (phyB[Tyr361Phe] and phyB[Arg582Ala]) (Zhang et al., 2013) that are not sensitive to temperature changes for a range of different temperatures and light condition. Although they could not detect a straight correlation between temperature and nuclear body size for wildtype phyB, they observed a strong reduction in nuclear body size at temperatures higher than $20^{\circ} \mathrm{C}$. By using a mathematical model describing the relation between Pfr-Pfr levels and nuclear body size they could show independently of the spectroscopic measurements that high temperatures decrease the apparent phyB Pfr-Pfr amount. Mathematical modeling of growth responses mediated by phyB, temperature and phyB-independent pathways further revealed that phyB-mediated temperature effects contribute significantly to growth regulation thereby showing largest effects at low irradiances (Legris et al., 2016).

Taken together, these studies support the idea that phyB is physiologically responsive to perceive light and temperature signals at the same time indicating that phy, in its active $\mathrm{Pfr}$ conformation, should also be defined as a temperature sensor. 


\section{CONCLUDING REMARKS}

Phytochrome signalling is an extensively studied field of photobiology. After learning the basics of the receptors' photochemistry, we have greatly extended our knowledge about the molecular mechanisms of phytochrome action, with a special respect to the identification of phytochrome-interacting protein partners. More recent findings revealed the molecular machinery that mediates integration of phytochrome signalling not only with hormoneinduced actions (de Lucas \& Prat, 2014; de Wit et al., 2016), but also those induced by various biotic and abiotic stresses (Ballare, 2014; Cortes et al., 2016) and by temperature (Jung et al., 2016; Legris et al., 2016). It is predictable that this trend will continue; however this review demonstrates that we still have a lot to learn about the phytochrome photoreceptors themselves.

\section{ACKNOWLEDGMENTS}

We sincerely apologize to all those colleagues whose important work is not cited because of space considerations.

The work in Szeged, Hungary was supported by two Hungarian Scientific Research Fund grants (OTKA, K-108559 and NN-110636) and grants from the Gazdaságfejlesztési és Innovációs Operatív Program (GINOP-2.3.2-15-2016-00015 and GINOP-2.3.2-15-201600001) to F.N.. Work in Edinburgh, UK was supported by a BBSRC grant BB/K006975/1 to F.N. 


\section{REFERENCES}

Ádám É, Hussong A, Bindics J, Wüst F, Viczián A, Essing M, Medzihradszky M, Kircher S, Schäfer E, Nagy F. 2011. Altered dark- and photoconversion of phytochrome B mediate extreme light sensitivity and loss of photoreversibility of the phyB-401 mutant. PLoS One 6(11): e27250.

Adam E, Kircher S, Liu P, Merai Z, Gonzalez-Schain N, Horner M, Viczian A, Monte E, Sharrock RA, Schafer E, et al. 2013. Comparative functional analysis of fulllength and N-terminal fragments of phytochrome C, D and E in red light-induced signaling. New Phytol 200(1): 86-96.

Al-Sady B, Ni W, Kircher S, Schäfer E, Quail PH. 2006. Photoactivated phytochrome induces rapid PIF3 phosphorylation prior to proteasome-mediated degradation. Molecular cell 23(3): 439-446.

Bae G, Choi G. 2008. Decoding of light signals by plant phytochromes and their interacting proteins. Annu Rev Plant Biol 59: 281-311.

Ballare CL. 2009. Illuminated behaviour: phytochrome as a key regulator of light foraging and plant anti-herbivore defence. Plant Cell Environ 32(6): 713-725.

Ballare CL. 2014. Light regulation of plant defense. Annu Rev Plant Biol 65: 335-363.

Brockmann J, Rieble S, Kazarinova-Fukshansky N, Seyfried M, Schäfer E. 1987. Phytochrome behaves as a dimer in vivo. Plant, Cell \& Environment 10(2): 105-111.

Burgie ES, Bussell AN, Walker JM, Dubiel K, Vierstra RD. 2014. Crystal structure of the photosensing module from a red/far-red light-absorbing plant phytochrome. Proceedings of the National Academy of Sciences of the United States of America 111(28): 10179-10184.

Carvalho RF, Campos ML, Azevedo RA. 2011. The role of phytochrome in stress tolerance. J Integr Plant Biol 53(12): 920-929.

Casal JJ. 2012. Shade avoidance. Arabidopsis Book 10: e0157.

Casal JJ. 2013. Photoreceptor signaling networks in plant responses to shade. Annu Rev Plant Biol 64: 403-427.

Casal JJ, Yanovsky MJ, Luppi JP. 2000. Two photobiological pathways of phytochrome A activity, only one of which shows dominant negative suppression by phytochrome B. Photochem Photobiol 71(4): 481-486.

Casson SA, Hetherington AM. 2014. phytochrome B Is required for light-mediated systemic control of stomatal development. Curr Biol 24(11): 1216-1221. 
Chen F, Li B, Li G, Charron J-B, Dai M, Shi X, Deng XW. 2014. Arabidopsis Phytochrome A Directly Targets Numerous Promoters for Individualized Modulation of Genes in a Wide Range of Pathways. The Plant cell 26(5): 1949-1966.

Chen M, Galvao RM, Li M, Burger B, Bugea J, Bolado J, Chory J. 2010. Arabidopsis HEMERA/pTAC12 initiates photomorphogenesis by phytochromes. Cell 141(7): 1230-1240.

Chen M, Schwab R, Chory J. 2003. Characterization of the requirements for localization of phytochrome B to nuclear bodies. Proceedings of the National Academy of Sciences of the United States of America 100(24): 14493-14498.

Chen M, Tao Y, Lim J, Shaw A, Chory J. 2005. Regulation of phytochrome B nuclear localization through light-dependent unmasking of nuclear-localization signals. Current biology : CB 15(7): 637-642.

Christians MJ, Gingerich DJ, Hua Z, Lauer TD, Vierstra RD. 2012. The light-response BTB1 and BTB2 proteins assemble nuclear ubiquitin ligases that modify phytochrome B and D signaling in Arabidopsis. Plant Physiology 160(1): 118-134.

Christie JM. 2007. Phototropin blue-light receptors. Annu Rev Plant Biol 58: 21-45.

Clack T, Mathews S, Sharrock RA. 1994. The phytochrome apoprotein family in Arabidopsis is encoded by five genes: the sequences and expression of PHYD and PHYE. Plant Mol Biol 25(3): 413-427.

Clack T, Shokry A, Moffet M, Liu P, Faul M, Sharrock RA. 2009. Obligate heterodimerization of Arabidopsis phytochromes $\mathrm{C}$ and $\mathrm{E}$ and interaction with the PIF3 basic helix-loop-helix transcription factor. Plant Cell 21(3): 786-799.

Cortes LE, Weldegergis BT, Boccalandro HE, Dicke M, Ballare CL. 2016. Trading direct for indirect defense? Phytochrome B inactivation in tomato attenuates direct antiherbivore defenses whilst enhancing volatile-mediated attraction of predators. New Phytol. 212: 1057-1071. doi:10.1111/nph.14210

de Lucas M, Prat S. 2014. PIFs get BRright: PHYTOCHROME INTERACTING FACTORs as integrators of light and hormonal signals. New Phytol 202(4): 11261141.

de Wit M, Galvao VC, Fankhauser C. 2016. Light-Mediated Hormonal Regulation of Plant Growth and Development. Annu Rev Plant Biol 67: 513-537.

Dechaine JM, Gardner G, Weinig C. 2009. Phytochromes differentially regulate seed germination responses to light quality and temperature cues during seed maturation. Plant Cell Environ 32(10): 1297-1309.

This article is protected by copyright. All rights reserved. 
Devlin PF, Kay SA. 2000. Cryptochromes are required for phytochrome signaling to the circadian clock but not for rhythmicity. Plant Cell 12(12): 2499-2510.

Duek PD, Fankhauser C. 2005. bHLH class transcription factors take centre stage in phytochrome signalling. Trends in Plant Science 10(2): 51-54.

Eichenberg K, Bäurle I, Paulo N, Sharrock RA, Rüdiger W, Schäfer E. 2000. Arabidopsis phytochromes $\mathrm{C}$ and $\mathrm{E}$ have different spectral characteristics from those of phytochromes A and B. FEBS letters 470(2): 107-112.

Elich TD, Chory J. 1997. Biochemical characterization of Arabidopsis wild-type and mutant phytochrome B holoproteins. The Plant cell 9(12): 2271-2280.

Elrouby N, Coupland G. 2010. Proteome-wide screens for small ubiquitin-like modifier (SUMO) substrates identify Arabidopsis proteins implicated in diverse biological processes. Proc Natl Acad Sci U S A 107(40): 17415-17420.

Endo M, Tanigawa Y, Murakami T, Araki T, Nagatani A. 2013. PHYTOCHROMEDEPENDENT LATE-FLOWERING accelerates flowering through physical interactions with phytochrome B and CONSTANS. Proc Natl Acad Sci U S A 110(44): 18017-18022.

Fankhauser C, Chen M. 2008. Transposing phytochrome into the nucleus. Trends in Plant Science 13(11): 596-601.

Fankhauser C, Yeh KC, Lagarias JC, Zhang H, Elich TD, Chory J. 1999. PKS1, a substrate phosphorylated by phytochrome that modulates light signaling in Arabidopsis. Science 284(5419): 1539-1541.

Finlayson SA, Krishnareddy SR, Kebrom TH, Casal JJ. 2010. Phytochrome regulation of branching in Arabidopsis. Plant Physiol 152(4): 1914-1927.

Franklin KA, Davis SJ, Stoddart WM, Vierstra RD, Whitelam GC. 2003. Mutant analyses define multiple roles for phytochrome $\mathrm{C}$ in Arabidopsis photomorphogenesis. Plant Cell 15(9): 1981-1989.

Franklin KA, Quail PH. 2010. Phytochrome functions in Arabidopsis development. $J$ Exp Bot 61(1): 11-24.

Franklin KA, Toledo-Ortiz G, Pyott DE, Halliday KJ. 2014. Interaction of light and temperature signalling. J Exp Bot 65(11): 2859-2871.

Fraser DP, Hayes S, Franklin KA. 2016. Photoreceptor crosstalk in shade avoidance. Curr Opin Plant Biol 33: 1-7. 
Gonzalez CV, Ibarra SE, Piccoli PN, Botto JF, Boccalandro HE. 2012. Phytochrome B increases drought tolerance by enhancing ABA sensitivity in Arabidopsis thaliana. Plant Cell Environ 35(11): 1958-1968.

Greenham K, McClung CR. 2015. Integrating circadian dynamics with physiological processes in plants. Nat Rev Genet 16(10): 598-610.

Hajdu A, Adam E, Sheerin DJ, Dobos O, Bernula P, Hiltbrunner A, Kozma-Bognar L, Nagy F. 2015. High-level expression and phosphorylation of phytochrome B modulates flowering time in Arabidopsis. Plant J 83(5): 794-805.

Han YJ, Kim HS, Kim YM, Shin AY, Lee SS, Bhoo SH, Song PS, Kim JI. 2010. Functional characterization of phytochrome autophosphorylation in plant light signaling. Plant Cell Physiol 51(4): 596-609.

Hennig L, Poppe C, Sweere U, Martin A, Schafer E. 2001. Negative interference of endogenous phytochrome B with phytochrome A function in Arabidopsis. Plant Physiol 125(2): 1036-1044.

Hennig L, Schafer E. 2001. Both subunits of the dimeric plant photoreceptor phytochrome require chromophore for stability of the far-red light-absorbing form. J Biol Chem 276(11): 7913-7918.

Hennig L, Stoddart WM, Dieterle M, Whitelam GC, Schafer E. 2002. Phytochrome E controls light-induced germination of Arabidopsis. Plant Physiol 128(1): 194-200.

Hickey CM, Wilson NR, Hochstrasser M. 2012. Function and regulation of SUMO proteases. Nat Rev Mol Cell Biol 13(12): 755-766.

Hiltbrunner A, Tscheuschler A, Viczián A, Kunkel T, Kircher S, Schäfer E. 2006. FHY1 and FHL act together to mediate nuclear accumulation of the phytochrome A photoreceptor. Plant \& cell physiology 47(8): 1023-1034.

Hirschfeld M, Tepperman JM, Clack T, Quail PH, Sharrock RA. 1998. Coordination of phytochrome levels in phyB mutants of Arabidopsis as revealed by apoproteinspecific monoclonal antibodies. Genetics 149(2): 523-535.

Hopkins JA, Kiss JZ. 2012. Phototropism and gravitropism in transgenic lines of Arabidopsis altered in the phytochrome pathway. Physiol Plant 145(3): 461-473.

Huang H, Yoo CY, Bindbeutel R, Goldsworthy J, Tielking A, Alvarez S, Naldrett MJ, Evans BS, Chen M, Nusinow DA. 2016. PCH1 integrates circadian and lightsignaling pathways to control photoperiod-responsive growth in Arabidopsis. eLife 5: e13292. 
Igamberdiev AU, Eprintsev AT, Fedorin DN, Popov VN. 2014. Phytochrome-mediated regulation of plant respiration and photorespiration. Plant Cell Environ 37(2): 290299.

Jang IC, Henriques R, Seo HS, Nagatani A, Chua NH. 2010. Arabidopsis PHYTOCHROME INTERACTING FACTOR proteins promote phytochrome B polyubiquitination by COP1 E3 ligase in the nucleus. Plant Cell 22(7): 2370-2383.

Jenkins GI. 2014. The UV-B photoreceptor UVR8: from structure to physiology. Plant Cell 26(1): 21-37.

Jiang Z, Xu G, Jing Y, Tang W, Lin R. 2016. Phytochrome B and REVEILLE1/2-mediated signalling controls seed dormancy and germination in Arabidopsis. Nat Commun 7: 12377 .

Johansson H, Jones HJ, Foreman J, Hemsted JR, Stewart K, Grima R, Halliday KJ. 2014. Arabidopsis cell expansion is controlled by a photothermal switch. Nat Commun 5: 4848.

Jung JH, Domijan M, Klose C, Biswas S, Ezer D, Gao M, Khattak AK, Box MS, Charoensawan V, Cortijo S, et al. 2016. Phytochromes function as thermosensors in Arabidopsis. Science 354(6314): 886-889.

Kami C, Lorrain S, Hornitschek P, Fankhauser C. 2010. Light-regulated plant growth and development. Curr Top Dev Biol 91: 29-66.

Kim J, Song K, Park E, Kim K, Bae G, Choi G. 2016. Epidermal Phytochrome B Inhibits Hypocotyl Negative Gravitropism Non-Cell Autonomously. Plant Cell.

Kim JI, Shen Y, Han YJ, Park JE, Kirchenbauer D, Soh MS, Nagy F, Schafer E, Song PS. 2004. Phytochrome phosphorylation modulates light signaling by influencing the protein-protein interaction. Plant Cell 16(10): 2629-2640.

Kim K, Shin J, Lee SH, Kweon HS, Maloof JN, Choi G. 2011. Phytochromes inhibit hypocotyl negative gravitropism by regulating the development of endodermal amyloplasts through phytochrome-interacting factors. Proc Natl Acad Sci USA 108(4): 1729-1734.

Kim W-Y, Fujiwara S, Suh S-S, Kim J, Kim Y, Han L, David K, Putterill J, Nam HG, Somers DE. 2007. ZEITLUPE is a circadian photoreceptor stabilized by GIGANTEA in blue light. Nature 449(7160): 356-360.

Klose C, Venezia F, Hussong A, Kircher S, Schafer E, Fleck C. 2015a. Systematic analysis of how phytochrome B dimerization determines its specificity. Nature Plants 1: 15090 . 
Klose C, Viczian A, Kircher S, Schafer E, Nagy F. 2015b. Molecular mechanisms for mediating light-dependent nucleo/cytoplasmic partitioning of phytochrome photoreceptors. New Phytol 206(3): 965-971.

Koini MA, Alvey L, Allen T, Tilley CA, Harberd NP, Whitelam GC, Franklin KA. 2009. High temperature-mediated adaptations in plant architecture require the bHLH transcription factor PIF4. Curr Biol 19(5): 408-413.

Kretsch T, Poppe C, Schäfer E. 2000. A new type of mutation in the plant photoreceptor phytochrome B causes loss of photoreversibility and an extremely enhanced light sensitivity. The Plant journal : for cell and molecular biology 22(3): 177-186.

Lagarias JC, Mercurio FM. 1985. Structure function studies on phytochrome. Identification of light-induced conformational changes in 124-kDa Avena phytochrome in vitro. $J$ Biol Chem 260(4): 2415-2423.

Lee KP, Piskurewicz U, Tureckova V, Carat S, Chappuis R, Strnad M, Fankhauser C, Lopez-Molina L. 2012. Spatially and genetically distinct control of seed germination by phytochromes A and B. Genes Dev 26(17): 1984-1996.

Legris M, Klose C, Burgie ES, Rojas CC, Neme M, Hiltbrunner A, Wigge PA, Schafer E, Vierstra RD, Casal JJ. 2016. Phytochrome B integrates light and temperature signals in Arabidopsis. Science 354(6314): 897-900.

Leivar P, Monte E, Oka Y, Liu T, Carle C, Castillon A, Huq E, Quail PH. 2008. Multiple phytochrome-interacting bHLH transcription factors repress premature seedling photomorphogenesis in darkness. Current biology : CB 18(23): 1815-1823.

Leivar P, Quail PH. 2011. PIFs: pivotal components in a cellular signaling hub. Trends in Plant Science 16(1): 19-28.

Liu P, Sharrock RA. 2013. Directed dimerization: an in vivo expression system for functional studies of type II phytochromes. Plant J 75(6): 915-926.

Lorrain S, Allen T, Duek PD, Whitelam GC, Fankhauser C. 2008. Phytochromemediated inhibition of shade avoidance involves degradation of growth-promoting bHLH transcription factors. Plant J 53(2): 312-323.

Lu X-D, Zhou C-M, Xu P-B, Luo Q, Lian H-L, Yang H-Q. 2015. Red-light-dependent interaction of phyB with SPA1 promotes COP1-SPA1 dissociation and photomorphogenic development in Arabidopsis. Molecular plant 8(3): 467-478.

Mancinelli AL 1994. The physiology of phytochrome action. In: Kendrick RE, Kronenberg GHM eds. Photomorphogenesis in Plants. Dordrecht: Springer Netherlands, 211-269. 
Martinez-Garcia JF, Huq E, Quail PH. 2000. Direct targeting of light signals to a promoter element-bound transcription factor. Science (New York, N.Y.) 288(5467): 859-863.

Mas P, Kim WY, Somers DE, Kay SA. 2003. Targeted degradation of TOC1 by ZTL modulates circadian function in Arabidopsis thaliana. Nature 426(6966): 567-570.

Matsushita T, Mochizuki N, Nagatani A. 2003. Dimers of the N-terminal domain of phytochrome B are functional in the nucleus. Nature 424(6948): 571-574.

McNellis TW, Deng XW. 1995. Light control of seedling morphogenetic pattern. Plant Cell 7(11): 1749-1761.

Medzihradszky M, Bindics J, Ádám É, Viczián A, Klement É, Lorrain S, Gyula P, Mérai Z, Fankhauser C, Medzihradszky KF, et al. 2013. Phosphorylation of phytochrome B inhibits light-induced signaling via accelerated dark reversion in Arabidopsis. The Plant cell 25(2): 535-544.

Miller MJ, Barrett-Wilt GA, Hua Z, Vierstra RD. 2010. Proteomic analyses identify a diverse array of nuclear processes affected by small ubiquitin-like modifier conjugation in Arabidopsis. Proc Natl Acad Sci U S A 107(38): 16512-16517.

Miura K, Hasegawa PM. 2010. Sumoylation and other ubiquitin-like post-translational modifications in plants. Trends Cell Biol 20(4): 223-232.

Nagatani A. 2010. Phytochrome: structural basis for its functions. Curr Opin Plant Biol 13(5): 565-570.

Nagy F, Schafer E. 2002. Phytochromes control photomorphogenesis by differentially regulated, interacting signaling pathways in higher plants. Annu Rev Plant Biol 53: 329-355.

Ni M, Tepperman JM, Quail PH. 1998. PIF3, a phytochrome-interacting factor necessary for normal photoinduced signal transduction, is a novel basic helix-loop-helix protein. Cell 95(5): 657-667.

Ni M, Tepperman JM, Quail PH. 1999. Binding of phytochrome B to its nuclear signalling partner PIF3 is reversibly induced by light. Nature 400(6746): 781-784.

Ni W, Xu S-L, Chalkley RJ, Pham TND, Guan S, Maltby DA, Burlingame AL, Wang ZY, Quail PH. 2013. Multisite light-induced phosphorylation of the transcription factor PIF3 is necessary for both its rapid degradation and concomitant negative feedback modulation of photoreceptor phyB levels in Arabidopsis. The Plant cell 25(7): 2679-2698. 
Ni W, Xu SL, Tepperman JM, Stanley DJ, Maltby DA, Gross JD, Burlingame AL, Wang ZY, Quail PH. 2014. A mutually assured destruction mechanism attenuates light signaling in Arabidopsis. Science 344(6188): 1160-1164.

Nito K, Wong CC, Yates JR, 3rd, Chory J. 2013. Tyrosine phosphorylation regulates the activity of phytochrome photoreceptors. Cell Rep 3(6): 1970-1979.

Novatchkova M, Tomanov K, Hofmann K, Stuible HP, Bachmair A. 2012. Update on sumoylation: defining core components of the plant SUMO conjugation system by phylogenetic comparison. New Phytol 195(1): 23-31.

Nozue K, Covington MF, Duek PD, Lorrain S, Fankhauser C, Harmer SL, Maloof JN. 2007. Rhythmic growth explained by coincidence between internal and external cues. Nature 448(7151): 358-361.

Nusinow DA, Helfer A, Hamilton EE, King JJ, Imaizumi T, Schultz TF, Farre EM, Kay SA. 2011. The ELF4-ELF3-LUX complex links the circadian clock to diurnal control of hypocotyl growth. Nature 475(7356): 398-402.

Oka Y, Matsushita T, Mochizuki N, Quail PH, Nagatani A. 2008. Mutant screen distinguishes between residues necessary for light-signal perception and signal transfer by phytochrome B. PLoS genetics 4(8): e1000158.

Osterlund MT, Deng XW. 1998. Multiple photoreceptors mediate the light-induced reduction of GUS-COP1 from Arabidopsis hypocotyl nuclei. The Plant journal : for cell and molecular biology 16(2): 201-208.

Pacin M, Legris M, Casal JJ. 2014. Rapid decline in nuclear costitutive photomorphogenesis 1 abundance anticipates the stabilization of its target elongated hypocotyl5 in the light. Plant Physiology 164(3): 1134-1138.

Park E, Park J, Kim J, Nagatani A, Lagarias JC, Choi G. 2012. Phytochrome B inhibits binding of phytochrome-interacting factors to their target promoters. The Plant journal : for cell and molecular biology 72(4): 537-546.

Pfeiffer A, Nagel M-K, Popp C, Wüst F, Bindics J, Viczián A, Hiltbrunner A, Nagy F, Kunkel T, Schäfer E. 2012. Interaction with plant transcription factors can mediate nuclear import of phytochrome B. Proceedings of the National Academy of Sciences of the United States of America 109(15): 5892-5897.

Phee BK, Kim JI, Shin DH, Yoo J, Park KJ, Han YJ, Kwon YK, Cho MH, Jeon JS, Bhoo SH, et al. 2008. A novel protein phosphatase indirectly regulates phytochromeinteracting factor 3 via phytochrome. Biochem J 415(2): 247-255. 
Quint M, Delker C, Franklin KA, Wigge PA, Halliday KJ, van Zanten M. 2016. Molecular and genetic control of plant thermomorphogenesis. Nat Plants 2: 15190.

Rausenberger J, Hussong A, Kircher S, Kirchenbauer D, Timmer J, Nagy F, Schäfer E, Fleck C. 2010. An integrative model for phytochrome B mediated photomorphogenesis: from protein dynamics to physiology. PLoS One 5(5): e10721.

Rockwell NC, Su YS, Lagarias JC. 2006. Phytochrome structure and signaling mechanisms. Annu Rev Plant Biol 57: 837-858.

Ryu JS, Kim JI, Kunkel T, Kim BC, Cho DS, Hong SH, Kim SH, Fernandez AP, Kim Y, Alonso JM, et al. 2005. Phytochrome-specific type 5 phosphatase controls light signal flux by enhancing phytochrome stability and affinity for a signal transducer. Cell 120(3): 395-406.

Sadanandom A, Adam E, Orosa B, Viczian A, Klose C, Zhang C, Josse EM, KozmaBognar L, Nagy F. 2015. SUMOylation of phytochrome-B negatively regulates light-induced signaling in Arabidopsis thaliana. Proc Natl Acad Sci U S A 112(35): 11108-11113.

Sakamoto K, Nagatani A. 1996. Nuclear localization activity of phytochrome B. The Plant journal : for cell and molecular biology 10(5): 859-868.

Sanchez-Lamas M, Lorenzo CD, Cerdan PD. 2016. Bottom-up Assembly of the Phytochrome Network. PLoS Genet 12(11): e1006413.

Schäfer E, Schmidt W. 1974. Temperature dependence of phytochrome dark reactions. Planta 116(3): 257-266.

Schneider-Poetsch HA, Braun B, Marx S, Schaumburg A. 1991. Phytochromes and bacterial sensor proteins are related by structural and functional homologies. Hypothesis on phytochrome-mediated signal-transduction. FEBS Lett 281(1-2): 245249.

Seo HS, Yang J-Y, Ishikawa M, Bolle C, Ballesteros ML, Chua N-H. 2003. LAF1 ubiquitination by COP1 controls photomorphogenesis and is stimulated by SPA1. Nature 423(6943): 995-999.

Sharrock RA, Clack T. 2002. Patterns of expression and normalized levels of the five Arabidopsis phytochromes. Plant Physiol 130(1): 442-456.

Sharrock RA, Clack T. 2004. Heterodimerization of type II phytochromes in Arabidopsis. Proc Natl Acad Sci U S A 101(31): 11500-11505.

Sheerin DJ, Menon C, zur Oven-Krockhaus S, Enderle B, Zhu L, Johnen P, Schleifenbaum F, Stierhof YD, Huq E, Hiltbrunner A. 2015. Light-activated 
phytochrome A and B interact with members of the SPA family to promote photomorphogenesis in Arabidopsis by reorganizing the COP1/SPA complex. Plant Cell 27(1): 189-201.

Shen H, Zhu L, Castillon A, Majee M, Downie B, Huq E. 2008. Light-induced phosphorylation and degradation of the negative regulator PHYTOCHROMEINTERACTING FACTOR1 from Arabidopsis depend upon its direct physical interactions with photoactivated phytochromes. The Plant cell 20(6): 1586-1602.

Shen Y, Khanna R, Carle CM, Quail PH. 2007. Phytochrome induces rapid PIF5 phosphorylation and degradation in response to red-light activation. Plant Physiology 145(3): 1043-1051.

Shin A-Y, Han Y-J, Baek A, Ahn T, Kim SY, Nguyen TS, Son M, Lee KW, Shen Y, Song P-S, et al. 2016. Evidence that phytochrome functions as a protein kinase in plant light signalling. Nature communications 7: 11545.

Shin J, Kim K, Kang H, Zulfugarov IS, Bae G, Lee C-H, Lee D, Choi G. 2009. Phytochromes promote seedling light responses by inhibiting four negatively-acting phytochrome-interacting factors. Proceedings of the National Academy of Sciences of the United States of America 106(18): 7660-7665.

Shinomura T, Nagatani A, Hanzawa H, Kubota M, Watanabe M, Furuya M. 1996. Action spectra for phytochrome A- and B-specific photoinduction of seed germination in Arabidopsis thaliana. Proc Natl Acad Sci U S A 93(15): 8129-8133.

Short TW. 1999. Overexpression of Arabidopsis phytochrome B inhibits phytochrome A function in the presence of sucrose. Plant Physiol 119(4): 1497-1506.

Su L, Hou P, Song M, Zheng X, Guo L, Xiao Y, Yan L, Li W, Yang J. 2015. Synergistic and Antagonistic Action of Phytochrome (Phy) A and PhyB during Seedling DeEtiolation in Arabidopsis thaliana. Int J Mol Sci 16(6): 12199-12212.

Subramanian C, Kim B-H, Lyssenko NN, Xu X, Johnson CH, Arnim AGv. 2004. The Arabidopsis repressor of light signaling, COP1, is regulated by nuclear exclusion: mutational analysis by bioluminescence resonance energy transfer. Proceedings of the National Academy of Sciences of the United States of America 101(17): 6798-6802.

Sweere U, Eichenberg K, Lohrmann J, Mira-Rodado V, Baurle I, Kudla J, Nagy F, Schafer E, Harter K. 2001. Interaction of the response regulator ARR4 with phytochrome B in modulating red light signaling. Science 294(5544): 1108-1111. 
Takala H, Björling A, Berntsson O, Lehtivuori H, Niebling S, Hoernke M, Kosheleva I, Henning R, Menzel A, Ihalainen JA, et al. 2014. Signal amplification and transduction in phytochrome photosensors. Nature 509(7499): 245-248.

Trupkin SA, Legris M, Buchovsky AS, Tolava Rivero MB, Casal JJ. 2014. Phytochrome B Nuclear Bodies Respond to the Low Red to Far-Red Ratio and to the Reduced Irradiance of Canopy Shade in Arabidopsis. Plant Physiology 165(4): 1698-1708.

Valverde F, Mouradov A, Soppe W, Ravenscroft D, Samach A, Coupland G. 2004. Photoreceptor regulation of CONSTANS protein in photoperiodic flowering. Science 303(5660): 1003-1006.

van Buskirk EK, Reddy AK, Nagatani A, Chen M. 2014. Photobody Localization of Phytochrome B Is Tightly Correlated with Prolonged and Light-Dependent Inhibition of Hypocotyl Elongation in the Dark. Plant Physiology 165(2): 595-607.

Vierstra RD, Zhang J. 2011. Phytochrome signaling: solving the Gordian knot with microbial relatives. Trends Plant Sci 16(8): 417-426.

Wagner D, Koloszvari M, Quail PH. 1996. Two Small Spatially Distinct Regions of Phytochrome B Are Required for Efficient Signaling Rates. Plant Cell 8(5): 859-871.

Wagner D, Tepperman JM, Quail PH. 1991. Overexpression of Phytochrome B Induces a Short Hypocotyl Phenotype in Transgenic Arabidopsis. The Plant cell 3(12): 12751288.

Wang FF, Lian HL, Kang CY, Yang HQ. 2010. Phytochrome B is involved in mediating red light-induced stomatal opening in Arabidopsis thaliana. Mol Plant 3(1): 246-259.

Xu X, Paik I, Zhu L, Bu Q, Huang X, Deng XW, Huq E. 2014. PHYTOCHROME INTERACTING FACTOR1 Enhances the E3 Ligase Activity of CONSTITUTIVE PHOTOMORPHOGENIC1 to Synergistically Repress Photomorphogenesis in Arabidopsis. Plant Cell 26(5): 1992-2006.

Yeh KC, Lagarias JC. 1998. Eukaryotic phytochromes: light-regulated serine/threonine protein kinases with histidine kinase ancestry. Proc Natl Acad Sci U S A 95(23): 13976-13981.

Yu X, Liu H, Klejnot J, Lin C. 2010. The Cryptochrome Blue Light Receptors. Arabidopsis Book 8: e0135.

Zhang J, Stankey RJ, Vierstra RD. 2013. Structure-guided engineering of plant phytochrome B with altered photochemistry and light signaling. Plant Physiology 161(3): 1445-1457. 
Zheng X, Wu S, Zhai H, Zhou P, Song M, Su L, Xi Y, Li Z, Cai Y, Meng F, et al. 2013. Arabidopsis phytochrome B promotes SPA1 nuclear accumulation to repress photomorphogenesis under far-red light. Plant Cell 25(1): 115-133.

Zhu L, Huq E. 2014. Suicidal co-degradation of the phytochrome interacting factor 3 and phytochrome B in response to light. Mol Plant 7(12): 1709-1711.

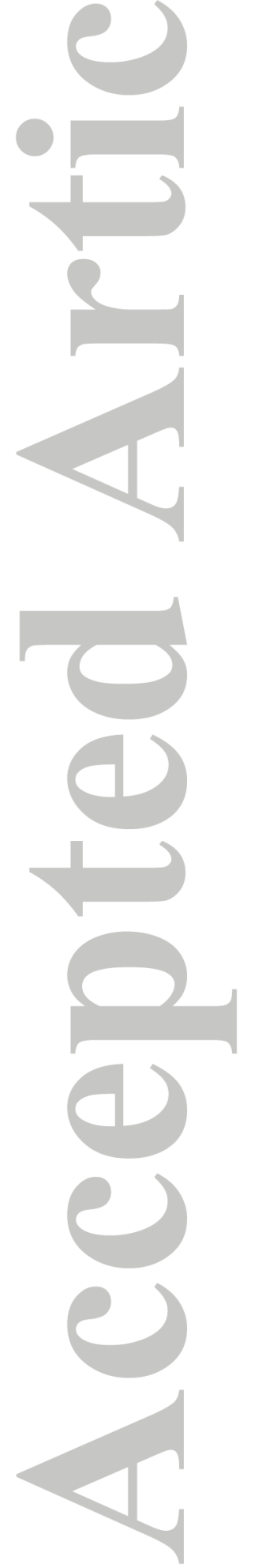


A

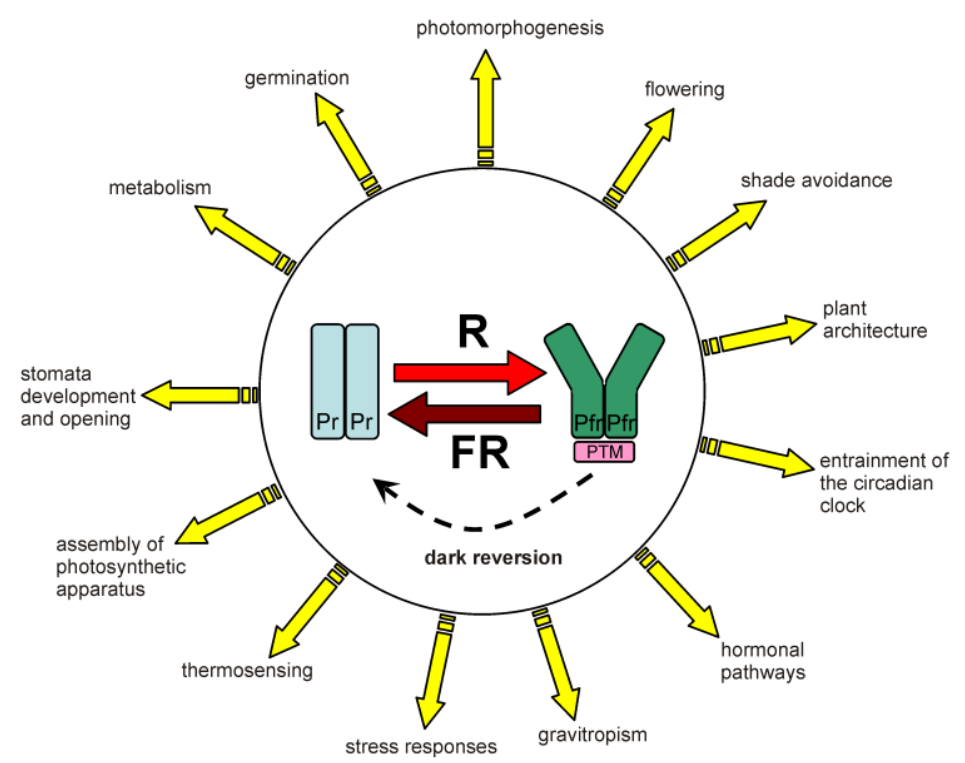

B

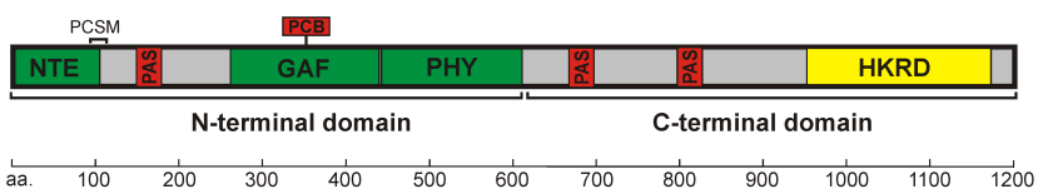

PCB phytochromobilin chromophore:

NTE: N-terminal extension

PCSM: Phosphorylation Cluster of Signaling Modulation

PAS: period-artn-singleminded domain

GAF: cGMP-specific phosphodiesterases, adenylyl cyclases and FhIA domain

PHY: phytochrome domain

HKRD: histidine kinase related domain

Figure 1. A. Phytochrome B-controlled responses in Arabidopsis thaliana.

The ratio of available Pr and Pfr forms of phyB molecules are tuned by the intensity of red (R) and far-red (FR) light (photoconversion) together with the dark reversion. The Pr/Pfr dimers are not depicted to maintain clarity (see text for details). PTM indicates posttranslational modifications of the Pfr form.

B. Schematic structure of the phyB monomer. 